\title{
A Reliable and Secure Smart Grid Communication Network Using a Comprehensive Cost Function
}

\author{
Vahid Kouhdaragh \\ Dept. Electrical, Electronic and Information Engineering, University of Bologna, Bologna 40136, Italy
}

Received: September 06, 2016 / Accepted: September 18, 2016 / Published: February 28, 2017.

\begin{abstract}
SG (smart grids) is an intelligent power grid in which the diverse nodes should communicate different types of information which have different communication requirements with CS (control stations). There exist several RATs (radio access technologies), with diversification in quality of service character which respect to the SG nodes communication requirements. On the other side, spectrum is becoming a rare source and its demands request is increasing exponentially. Therefore, resource allocation to support different types of SG nodes should be elaborated so that the resource efficiency is maximized while the SG communication requirements are respected. Using a CF (cost function) based on the SG node requirements and RATs characteristics to find the desirability value of every RATs for a certain node type accomplish this goal in combination with prioritizing the different SG nodes types based on SG goals by creating a priority table for RATs and different SG node types. The main node communication requirements are formulized to be used in the CF in this paper. The numerical results show that the proposed method defines the desirability value of each RAT for a certain SG node type that helps to make a priority table by using the SG node prioritization table.
\end{abstract}

Key words: Smart grid node prioritization, cost function, communication requirements, spectral efficiency, heterogeneous network.

\section{Introduction}

Due to this fact that the conventional power grids are no more efficient, it is required to bring in a new model to realize current needs in an efficient way, namely the SG (smart grids) [1-3]. Different types of SG nodes type are increasing. These node types report power information to the CS (control station) or CC (control center) through the aggregators. Then the information is analyzed and the appropriate actions and command are issued and then the demand responses sent back to the SG devices [3]. Each SG branch of nodes has different communication requirements. These types of SG nodes are considered as an application of M2M (machine to machine) communication that usually generates low rate traffic [3]. The collected data are usually transferred to the CS by using the RATs (radio access technologies) [3-5]. On the other hand, due to different SG nodes communication requirements,

Corresponding author: Vahid Kouhdaragh, Ph.D., research fields: telecommunications, wireless engineering. designing a SGCN (smart grid communication requirements) respect to the RATs characteristics get a significant subject $[1,3,6]$. Moreover, due to the increasing number of deployed SG nodes, the demand request increases which results in increasing spectrum resources usage [1-3]. Thus, spectrum scarcity problems results in an increased interest in the research area, especially when is applied to M2M application such as SGCN [4, 5, 7]. Therefore, it is considerably essential to find the appropriate solutions to allocate the limited wireless resources efficiently while SGCN requirements are fulfilled. The scope of this paper is focused to design a method and algorithm for properly allocating the communication resources of different RATs to the SG nodes that having different communication requirements, by making a priority table that shows the desirability of each RAT for a certain type of a user in combination of the SG node prioritization table $[2,3]$. We focus on all main $\mathrm{SG}$ node type communication requirements as the communication KPIs (key performance indicators), 
namely latency, data rate, reliability and security. On one hand, it is aimed at fulfilling a minimum required data rate, a maximum latency an acceptable reliability and security requirements for all SG node type while we focus on a solution that leads network designer to reduce the resource wasting by restricting the allocation of unnecessary resources to the SG nodes. In fact, it is more preferable that the nodes having lower latency sensitivity requirements being supported by RATs having higher intrinsic delays, e.g., GSM (high number of routers, high routing duration), so it results in leaving the RATs with lower delays for the nodes having a higher delay sensitivity while reliability and security requirements of the mentioned SG node type are fulfilled. Reliability of the RATs are measured by means of the MMPR (mismatch probability), in which the SG node type generating rate, RATs intrinsic latency and SG node delay sensitivity are its major parameters [8]. To this goal, a properly planned CF is proposed aiming at selecting the priority of each available RATs for the different SG nodes type. In the other words, the desirability value of different RATs supporting a certain node type is defined. Besides, the resources should be fairly divided among the same and different type of the node types based on the desirability of the mentioned RAT (achieving by using the $\mathrm{CF}$ ) for the certain type of the node. Putting all the eggs in one basket decreases the reliability of the system. Also offloaded extra traffic from a certain nodes can be assigned to the next high priority RAT [1-3]. Besides, the prioritization of SG nodes based on the main SG goals in combination of RATs desirability table for different SG node types help to elaborate an algorithm to have a priority table to allocate RATs spectrum to the different SG user types. The proposed method is very precious and efficient comparing to other methods because it has a low complexity while considering all node type communication requirements especially reliability and security to assess their fitting value with RATs communication characteristics. To the best of our knowledge, the other methods suggested in the literature are not efficient. Besides, there are no simple resource allocation methods for fulfilling the SGCN requirements with the given specific constraints. Moreover, some resource allocation methods have been done regardless of considering the efficient match between RATs communication characteristics and SG node type communication requirements [9-12]. Finally, an efficient resource allocation can be done by considering the SG node type priority in sense of SG goals. This issue has been considered in this paper. In the next sections, system models and proposed CF to define the desirability values, SG node prioritization and the proposed solution, numerical results and conclusion are discussed.

\section{Smart Grid Node Prioritization}

The prioritization of the SG nodes based on the SG goals allows selecting the most important nodes. The nodes having a higher priority in the SG may be served earlier. Therefore, it is needed to define the SG goals as the KPIs for finding the weight of the SG nodes. Then, it is needed to give a value to each different KPI for a certain node type. For a certain type of the SG nodes, giving more importance to a certain KPI depends on how much that node can fulfill that corresponding goal (KPI). The perceptive concept we propose is proportional to a quantitative value in sense of $\mathrm{SG}$ goal satisfaction, as follows: Very high: 5, High: 4, Medium: 3, Low: 2 and Very low: 1.

SG node prioritization effectiveness depends strongly on how much they can fulfill the SG goals. The main goals of SG have been declared in many references. They are considered as the KPIs for prioritization method [6]. In the following, a description of different types of SG goals and the services relying on the SGs are presented, and a qualitative prioritization is performed. At first, it is needed to identify the main goals of the SG as follows: green energy, reliability in power grid, security in power grid, outage avoidance, users cooperation, automated maintenance, consumption cost minimizing 
and disaster avoidance $[2,6]$.

Green energy concept in SG is generally defined as energy usage efficiency, decrease using of fossil fuels and try to use the sustainable energy. As a matter of fact, reliability in power grid, controlling part and distribution grid management is a main criterion. Increased reliance on renewable improve reliability in associated unpresented disasters [6]. As the technology develops, dependency on the secure electricity supplies, transmission and distribution part is increased. Grid monitoring and surveillances due to its characteristics has a significant role on SG security [2, 6]. Outages and blackouts avoidance as the result of high consumption or any unpresented faults in the power grid should be considered as an important goal of SG [2, 6]. User cooperation is considered as the users' assistance to increase power grid functionality on the demand side of the SG. It should be mentioned that power system status as the received information by CS changes demand responses based on its analysis [2, 6]. Automated maintenance is an intelligence system whose actions are started automatically at regular intervals to perform maintenance operations. To this aim, SG should monitor all critical components of the power grid $[2,6]$. Decreasing the consumption cost in the SG platform helps users schedule electrical appliance issues, minimizing variance in power consumption. SG demand side nodes have high effects on it. Although, power grid status controlling (distributing part) has effects on decreasing consumption cost by detecting the fault over the power grid $[2,6]$. Disaster avoidance is another important goal of SG which can be achieved through higher rates of survivability following a natural disaster. Besides, DGM by balancing the power distribution is helpful for disaster avoidances in power grid. SG's demand side role (by communicating with $\mathrm{CS}$ ) on disaster avoidance is so notable $[2,6]$.

\subsection{The SG Node Types and Node Prioritization}

There are various types of SG node types, each one with different function and communication requirements. In this part, the most important SG node types and their communication requirements are presented. AMI (advanced metering infrastructure) is a set of SMs, communications networks, and data managing systems, for facilitating and enabling SMs to have two-way communications with the CS $[2,6]$. The WASA (wide area situational awareness) nodes supervise the power system over a wide geographic area. Thus, WASA has the significant role in identifying SG status and surveillances issues. DERS (distributed energy resources) are used to make possible renewable energy resources as a main part of the future SG to integrate and unify them into the power grid infrastructure. Besides, DERS have the role as the power supply resources for urgent situation usage during outages and remarkable disasters. The PHEV (plug in electrical vehicle) is useful for reducing greenhouse gas emissions and fuel consumption. It is because they present the useful information of the device charger for PHEVs $[2,6]$. Additionally, node densities in SG do not have defined values.

The DGM (distributed grid management) entity allows utilities to distantly monitor and control the required power grid parameters in the $\mathrm{SG}$ distribution network $[2,6]$. Based on the nodes functions in the SG and the policy which were described above, allocating the numerical values to the nodes based on an intuitive understanding of their functions on fulfilling a certain SG goal is defined and the results can be shown in Table 1. To be clearer, the demand side nodes (AMI, PHEV and even DERS) are investigated here. Large number of users in AMI part, which includes the real users using SMs rather than PHEV, may cause to highlight importance of AMI to fulfill some SG goals rather than PHEV. Although, respect to the DERS characteristics, its important role to respect to SG goals are changed from the highest in green energy aspect and the lowest in automatic maintenance, Table $1[2,6]$. Moreover, distributed energy resources can fulfill user 
Table 1 SG goals and node prioritization.

\begin{tabular}{lllllll}
\hline & $\left(W_{\boldsymbol{g}}\right)$ & AMI & PHEV & WASA & DGM & DERS \\
\hline Green energy & 5 & 4 & 3 & 3 & 4 & 5 \\
Reliability & 4 & 3 & 3 & 5 & 5 & 3 \\
$\begin{array}{l}\text { Security } \\
\begin{array}{l}\text { Outage } \\
\text { avoidance }\end{array}\end{array}$ & 3.5 & 4 & 4 & 5 & 4 & 3 \\
$\begin{array}{l}\text { Users } \\
\text { cooperation }\end{array}$ & 3 & 5 & 2 & 5 & 4 & 4 \\
$\begin{array}{l}\text { Automated } \\
\text { maintenance }\end{array}$ & 3.5 & 1 & 1 & 5 & 3 & 1 \\
$\begin{array}{l}\text { Minimize } \\
\text { consumption }\end{array}$ & 4 & 5 & 4 & 3 & 2 & 3 \\
$\begin{array}{l}\text { Disaster } \\
\text { avoidance }\end{array}$ & 4.5 & 3 & 1 & 5 & 4 & 2 \\
$W N_{n}$ & - & 116 & 83 & 136 & 114 & 102 \\
$C F S G_{\max }$ & - & 0.85 & 0.61 & 1 & 0.84 & 0.75 \\
\hline
\end{tabular}

cooperation goals for the users who have access directly to it. The other sustainable energy resources can provide more power for the grid to avoid outages as well. By using the introduced references, which their summarized details are given, it can be shown clearly the rationale behind the numerical values in Table 1. Prioritization CF can be defined as Eq. (1).

$$
W N_{n}=\sum_{g=1}^{N_{\text {goal }}} W_{g} \cdot N_{n_{g}}
$$

in which $N_{\text {goal }}$ is the number of the goals of smart grid system, $W_{g}$ is the weight of the SG $g_{t h}$ goal (the goal importance based on the literature information and motivation to use SG $[4,6,12])$ and $N_{n_{g}}$ is the node $n$th normalized value (to fulfill the SG $g_{t h}$ goal). $W N_{n}$ is the non-normalized value of the cost function of node $n$ indicating degree of the importance of the node $n$ to fulfill all SG nodes. The CF is normalized to maximum value of $1, C F S G_{n}$, which is obtained from Eq. (2):

$$
C F S G_{n}=\frac{W N_{n}}{C F S G_{\max }}
$$

in which $C F S G_{\max }=\operatorname{maximum}\left\{W N_{1}, \ldots, W N_{n}\right\}$.

\section{System Model and Cost Function}

Defining a communication based CF to identify the best matched RAT and SG user in sense of RATs characteristics and SG node communication requirements is a proper method to evaluate the desirability value of each RAT which supports a certain SG node type $[1,2,13]$. The $\mathrm{CF}$ includes all communication parameters.

\subsection{KPIs and Nodes Communication Requirements}

Based on the definition in this paper, higher $\mathrm{CF}$ value dedicates on the lower desirability value and vice versa. Therefore, for a certain SG node type, the RATs desirability values are put in order, from highest priority to the lowest priority (from lowest $\mathrm{CF}$ value to the highest one). For the defined $\mathrm{CF}$, the main KPIs should be clarified and formulized [1, 2, 13]. As it was explained the main KPIs are data rate, delay, reliability and security. Main parts of the CF, namely weights and normalized values can be formulized in a way which higher weights or normalized values show the more undesirable matching and vice versa. The $\mathrm{CF}$ numerical value is restricted between 0 and $1 . \mathrm{SG}$ different node types are introduced in Section 2 and Table 2 gives a summary of the SG node types requirements, where the values have been defined by the UTC (Utilities Telecom Council), while in the last two column the values used in this paper are given. UTC has defined such communication requirements based on detailed studies for each SG application, by considering an average number of nodes and collectors per branch of the network users $[2,6]$. The generating rates, $\lambda_{i}$ of the nodes are given in Ref. [6] (i.e, WASA: $\lambda_{i}=0.1 \mathrm{~s}$, SMs: $\lambda_{i}=15 \mathrm{~min}$, PHEV: $\lambda_{i}=6 \mathrm{~h}$, DERS: $\lambda_{i}=4 \mathrm{~h}$ and DGM: $\lambda_{i}=1 \mathrm{~s}$ ).

\subsection{The Flowchart of the Introduced Method}

In this part the introduced method will be explained in general. Then the corresponding flowchart is shown in Fig. 1. At the first part the different SG node type and their communication requirements are defined. Their main communication requirements are considered as the KPIs. The number of different SG node type is $N$. The number of available RATs is $F$. At the first step the $i$ th SG node type is chosen and respect 
Table 2 Communication requirements of SG nodes.

\begin{tabular}{lllllll}
\hline & $\begin{array}{l}\text { Reference data rate } \\
(\mathrm{kb} / \mathrm{s})\end{array}$ & $\begin{array}{l}\text { Reference latency } \\
(\mathrm{s})\end{array}$ & Reliability & Security & $\begin{array}{l}\text { Selected data rate } \\
(\mathrm{kb} / \mathrm{s})\end{array}$ & Selected latency (s) \\
\hline AMI & 500 & $2-15$ & $99-99.99 \%$ & High & 500 & 2 \\
WASA & $600-1,500$ & $0.02-0.2$ & $99.999-99.9999 \%$ & High & 1,000 & 0.05 \\
DERS & $9.6-56$ & $0.02-15$ & $99-99.99 \%$ & High & 40 & 1 \\
PHEV & 100 & $2-300$ & $99-99.99 \%$ & Relatively high & 100 & 5 \\
DGM & $9.6-100$ & $0.1-2$ & $99-99.999 \%$ & High & 70 & 0.5 \\
\hline
\end{tabular}

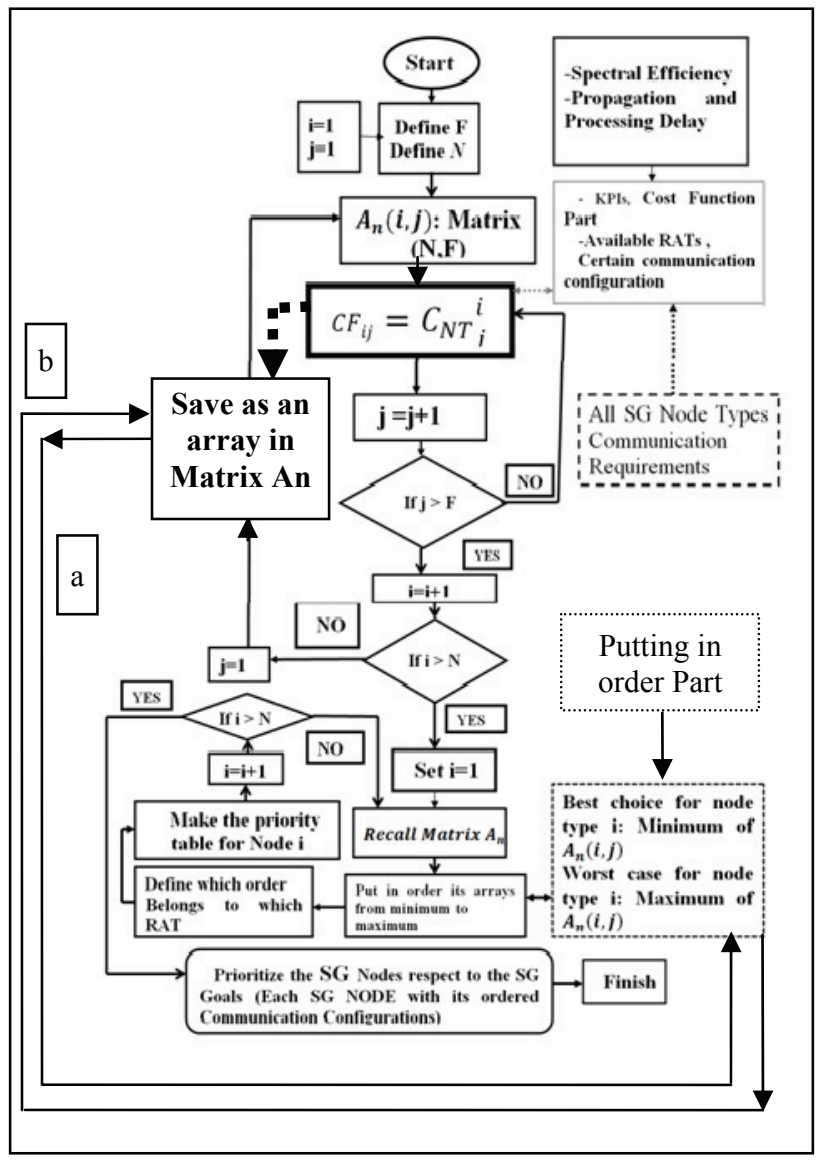

Fig. 1 Introduced method's flowchart.

to its communication requirements and $j$ th $\mathrm{RAT}$ characteristics, the CF is calculated, $C_{N T j}^{i}$, is put in $C F_{i j}$ and then it will repeated for all RATs ( $F$ number of RATs) and the results are put in the matrix arrays. Then the next SG user type is chosen and this process will be repeated till all SG node type CF for all RATs being calculated. At the next step the SG node prioritization is done and the SG nodes are put in order from higher priority to lower one, $A_{n}$. Putting in the order process is done in parallel (Line $\mathrm{a}$ and $\mathrm{b}$ in
Fig. 1) and at the end of calculating all $C F_{i j}$. At the final step for each certain SG, their highest priority RATs are put in order from higher to the lower ones. The RATs resource allocation can be done based on the achieved priority table and the SG network designer will have a great insight to design the SG communication network.

\subsection{The CF, Weights and Normalized Values}

Regarding to previous section, an assessment method namely $\mathrm{CF}$ is required for assessing the RATs desirability to support the different SG nodes types. In the other word, a suitable CF formula, Eq. (3), is introduced to manage the resource allocation policy for different nodes type with different communication requirements whose are supported by different RATs $[2,11,13]$. Any cognitive process for finding resources results in having delay that affects the KPIs values. As it was discussed, to reach to these targets, it is needed to determine the weights of the most important KPIs, and their normalized value when they are supported by different RATs. The most important KPIs are given in Table 3 which four main KPIs are given $[2,6]$.

For a certain scenario of a SG system including all types of SG node, the nodes having the lower data rate while fulfilling SG system goals, are more favorable than the other different type of the SG nodes with higher required data rate, thus have the lower weight and vice versa (Eq. (4.1)). To define the normalized value, a reference $\mathrm{BW}$ and the reference data rate,

Table 3 Communication requirements of SG nodes.

\begin{tabular}{l|ll}
\hline KPIs for SG nodes & Data rate Delay Reliability Security \\
\hline
\end{tabular} 
(data rate for a reference $\mathrm{BW}$ ) in each different RATs are considered. At the next step, the amount of data rate of a certain type of SG node is divided by each RATs data rate (for a certain BW in $\mathrm{Hz}$ ) (Eq. (4.2)). The same policy is applied to evaluate the weight for latency based on the delay sensitivity of each type of SG node (Eq. (5.1)). Thus, the nodes with lower delay sensitivity have lower weight and it is because that the nodes who can tolerate higher delay are more desirable comparing with the nodes who can tolerate lower delay.
A basic CF to reach to the goals is obtained by Eq. (3) as a result of Ref. [13].

$$
C F_{i j}=\left(\frac{1}{\sum_{q=1}^{N_{K P I}} W_{i q}} \cdot \sum_{q=1}^{N_{K P I_{u}}}\left(W_{i q} \cdot N_{q_{i j}}\right)\right)
$$

where, $N_{K P I_{u}}$ is the number of KPIs, $W_{i q}$ and $N_{q_{i j}}$ are the node $i$ weight, and Node $i$, RAT $j$ normalized values respectively, $i \in\{1, \ldots, N\}$ and $j \in\{1, \ldots, F\}$. Thus Eq. (3) can be rewritten as following:

$$
C F_{i j}=\frac{\left(W D R_{i} \cdot N D R_{i j}\right)+\left(W D E_{i} \cdot N D E_{i j}\right)+\left(W R E_{i} \cdot N R E_{i j}\right)+\left(W S E_{i} \cdot N S E_{i j}\right)}{\left(W D R_{i}+W D E_{i}+W R E_{i}+W S E_{i}\right)}
$$

where $C F_{i j}$ is the $C F$ value for the user type $i$ when using the RAT $j$, and $W_{D R_{i j}}$ and $N_{D R_{i j}}$, are the BW weight and normalized value for user type $i$ and RAT type $j$ correspondingly. $W_{\text {delay }_{i j}}$ and $N_{\text {delay }_{i j}}$, Eq. (5.2), are the delay weight and normalized value for user type $i$ supported by RAT type $j$, respectively. $W R E_{i}$ and $W S E_{i}$ are weights for reliability and security respectively. $N R E_{i j}$ and $N S E_{i j}$ are normalized value for reliability and security respectively as well. It should be mentioned that the considered $\mathrm{BW}$ for every RATs is $5 \mathrm{MHz}$. By using introduced method, the RATs with the latency closer to the delay sensitivity of SG nodes have lower delay normalized value and are more desirable in sense of delay matching between RAT latency and SG node delay requirement. The characteristics of the RATs selected for this study are in Table 4 [14-19]. Coding rates are assumed equals to 1 for all RATs configurations.

In Eq. (4.1), the BW weight for each node can be defined as

$$
W_{D R_{i j}}=R_{N i} / M
$$

where, $R_{N i}$ is the data rate required by the $i$ th node type, and $M$ equal to $\max \left\{R_{N 1}, R_{N 2}, \ldots, R_{N n}\right\}$ is maximum rate among all the $\mathrm{SG}$ node types. The desirability of RATs that support a certain type of SG nodes can be assessed by their $\mathrm{CF}$ value, where the lowest CF value has the highest priority. Consequently, the nodes with the lowest data rate have the lowest weight. The normalized BW value for the node $i$ in the network $j$ is

$$
N_{D R_{i j}}=\frac{R_{N i}}{P b p s N E T_{j}}
$$

where, $\operatorname{PbpSNET}_{j}$ is the proportional rate for certain fixed amount of BW. For example, $1 \mathrm{MHz}$ generates different data rate in different RATs, even in same RAT with different modulation scheme (e.g., 4 Mbps in LTE and 1.3 Mbps in GSM). The latency weight for node $i$ can be defined as:

$$
W_{\text {delay }_{i j}}=1-\frac{N W L A T_{i}}{M A X_{N L A T}}
$$

where $N W L A T_{i}$ is the maximum delay requirement for node $i$ (the last column of the Table 2) and $M A X_{N W L A T}$ is the maximum value among NWLAT . As mentioned before, the lowest $\mathrm{CF}$ value stands for a more efficient allocation. Thus, the node with the higher difference between the delay requirement and the latency of the allocated RAT has the higher weight. The normalized latency for node $i$, using the network $j$, can be defined as:

$$
N_{\text {delay }_{i j}}=1-\frac{\text { TotLat }_{i j}}{N W L A T_{i}}
$$

Table 4 RTT and SE for the RATs.

\begin{tabular}{lll}
\hline & RTT $(\mathrm{ms})$ & Spectrum efficiency $(\mathrm{bits} / \mathrm{s} / \mathrm{Hz})$ \\
\hline LTE & $10-20$ & $($ 64QAM modulation $) \simeq 3.6$ \\
GSM & $150-200$ & $($ GMSK modulation $) \simeq 1.36$ \\
$($ Satellite $)$ LEO & $100-150<$ & $($ 8PSK modulation $) \simeq 1.8$ \\
\hline
\end{tabular}


in which TotLat $i j$ is the total network latency (buffering, propagation, processing and framing size). The RATs having the latency higher than the maximum delay sensitivity of a certain SG node type are disregarded since they cannot fulfill the delay requirements of the SG node and, based on Eq. (5.2), the normalized value is negative. Since RTT is the reference value for the round trip time for each type of RATs and TP is the processing time, considered as 5 msec [20], it is referred into the following to Tot $L a t_{i j}$ equals to (RTT + TP), as the overall latency value. To be clearer, Fig. 2 shows the normalized delay as a function of RTT of RATs and the SG node delay sensitivity. As it shows, the normalized delay value is higher if RTT of the RAT and SG node delay sensitivity difference is higher and vice versa. This issue helps to define the KPI in a way that it would be desirable to allocate RATs with higher RTT to a lower delay sensitivity $\mathrm{SG}$ node type. Besides, it can be seen that whenever the RTT of a certain RATs is higher than SG node delay, its normalized value is negative and is excluded from the RATs to support the mentioned SG node type requirements. Also by considering any types of latency such as routing delay, the method can be used in the routers to choose the most efficient path to the CS. In case of using error correction, $N D_{i j, P E R}$ is the total data which should be resent then:

$$
N D_{i j, P E R}=\text { floor }\left(n p \times\left(\frac{N D_{i j}}{n_{g p}}+\alpha_{n c} \cdot P E R_{i j}\right)\right)
$$

In which $\alpha_{n c}$ is the percentage of the packets which have not been recovered and should be resent again, $n_{g p}$ is goodput, $N D_{i j}$ is the data buffered size of a node and $n p$ is the number of bits per packet.

The SG node type reliability weights, $W R E_{i}$, can be defined and weighted respect to their required reliability percentage, $W R i$, as following and it should be mentioned this values facilitate corresponding a numerical value to reliability weights Table 5 .

Mismatch probability, $M M P R_{i j}$, is the concept in which the RATs reliability can be evaluated for SG node type $i$, using RAT $j$. The concept includes a soft real time algorithm for model based context access configuration and QoS class assignment, and allows setting probabilistic bounds on the information reliability which is so-called mismatch probability, MMPR [8]. The ability of the RATs to adapt to the communicating nodes is often referred to as context awareness, and is becoming a key factor for users to be able to efficiently interact with applications and platforms in a highly dynamic world [8]. The reliability of the accessed information is the key to the success of any context aware applications, since application adaptation should respond to current events and not earlier ones. The node generating period, $\lambda_{i}$, is considered as the status changing in the

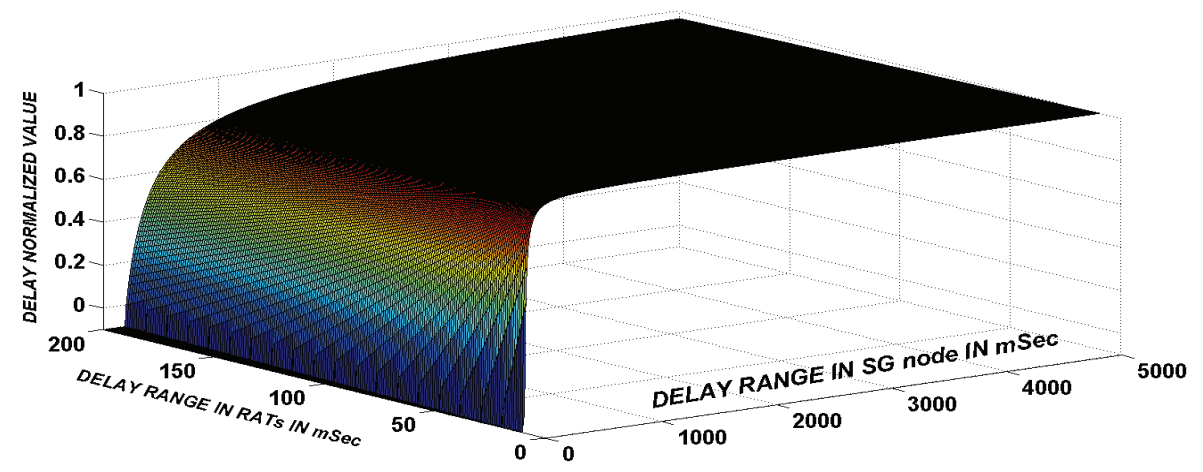

Fig. 2 Delay normalized value.

Table 5 Weight values table.

\begin{tabular}{llllll}
\hline$W R i \%$ & 1 & $1-0.99999$ & $0.99999-0.9999$ & $0.9999-0.999$ & $0.999-0.99$ \\
\hline$W R E_{i}$ & 1 & 0.8 & 0.6 & 0.4 & 0.2 \\
\hline
\end{tabular}


system. By considering reactive access scheme, meaning that the aggregator would send a request and get a response with the latest value, in its specific case (changing randomly very close to the $\lambda_{i}$, with exponential distributed time interval), it is applicable to compare reliability of RATs in any case. The delay sensitivity of the node is considered as the acceptable delay for MMPR and RAT latency is TotLat ${ }_{i j}$. MMPR has another parameter, Ploss $_{i j}$ which in literature is assumed as 0.01. MMPR can be acheived by Eq. (6) [8] and if the dealy $=N W L A T_{i}$ then the minimum MMPR can be acheived and the link utility or buffer size can be manupilated based on the network designer perspective.

$$
\begin{gathered}
M M P R_{i j}=1-\left(1-\text { Ploss }_{i j}\right)^{2} \times\left(\frac{\mathrm{v}_{i j}}{\lambda_{i}+\mathrm{v}_{i j}}\right), \\
\mathrm{v}_{i j}=\left(\frac{1}{\text { TotLat }_{i j}}\right)
\end{gathered}
$$

Higher MMPR causes higher normalized value for reliability those results in higher CF. Security in a network and assessing it is not a straightforward issue as the best of our knowledge there is no work to evaluate it for a certain type of the RATs. The fuzzy based or a simple CF to evaluate it based on the most important factors of security is an appropriate way to assess the different RATs security. Node's security non-numerical inception value can be mapped in the shape of numerical value to be evaluated. It is done as following (designer view).

$$
W S E_{i}=\left\{\begin{array}{l}
1 \text { if Node i security requirement is High } \\
0.8 \text { if Node i security requirement is Slightly high } \\
0.6 \text { if Node i security requirement is Medium } \\
0.4 \text { if Node i security requirement is Low } \\
0.2 \text { if Node i security requirement is Very low }
\end{array}\right.
$$

To evaluate the normalized value of each RAT it is needed to find out the main security issues in each RAT. Thus the main factors of secure RATs should be identified. Based on the literature, three main factors for a secure communication network com as following: encryption (ENP), complexity (COMC) and response time (RST), Tables 6 and 7 [21-27].

$N S E_{j}$ that is security non-normalized value for $\operatorname{RAT} j$ is:

$$
=\frac{\left(\propto_{E N} \cdot E N P_{j}+\propto_{C O M} \cdot C O M E_{j}+\propto_{R S T} \cdot R S T_{j}\right)}{\sum_{S E C=1}^{3} \propto_{S E C}}
$$

and then by making a set, $S E C$ using Eq. (7), then the normalized value for security is acheived from Eq. (7) and is shown in Table 8 and the normalize value to 1 is acheived by using Eq. (8). $\propto_{S E C}$ is the weight of each security KPIs, $\propto_{\mathrm{EN}}, \propto_{\mathrm{COM}}$ are encryption, complexity andresponse time weight respectively (here equals to 1 , depends to designer).

$$
\begin{gathered}
0<\propto_{\mathrm{SEC}}<1 \\
\mathrm{SEC}=\left\{\mathrm{NSE}_{1}, \mathrm{NSE}_{2}, \ldots, \mathrm{NSE}_{\mathrm{j}}\right\} \\
\left.\mathrm{Nsec}_{\text {max }}=\mathrm{Maximum} \mathrm{NSE}_{1}, \mathrm{NSE}_{2}, \ldots, \mathrm{NSE}_{\mathrm{j}}\right\} \\
\mathrm{NoSE}_{\mathrm{j}}=\frac{\mathrm{NSE}_{\mathrm{j}}}{\operatorname{Nsec}_{\max }}
\end{gathered}
$$

The RATs details are seen in Table 4. The values can be acheived by intuitively approach using Refs. [5, 21-26] rationaly.

Now SG nodes are prioritized based on the SG goals and all unknown parameters of CF are achieved and applied by using the CF formula, Eq. (3). The combination of them will be discussed in the numerical results. Additionally, the number of the same node type that can be synchronized in a time slot, $N_{\text {sync }}^{t s}$, for a certain RAT, can be achieved by using Eq. (9) as follows:

$$
\begin{aligned}
1 \leq N_{\text {sync }}^{t s} \leq \text { floor } & {\left[R B s_{\text {num }}^{M H z} \times t s_{\text {num }}\right.} \\
& \left.\times \frac{N W L A T_{i}}{D_{i}^{g}}\right]
\end{aligned}
$$

Table 6 RST normalized value.

\begin{tabular}{lllll}
\hline Very low & Low & Fairly high & High & Very high \\
\hline$\sim 1$ & $\sim 2$ & $\sim 3$ & $\sim 4$ & $\sim 5$ \\
\hline
\end{tabular}


Table 7 ENP and COMP normalized value.

\begin{tabular}{lllll}
\hline Very low & Low & Fairly high & High & Very high \\
\hline$\sim 5$ & $\sim 4$ & $\sim 3$ & $\sim 2$ & $\sim 1$ \\
\hline
\end{tabular}

Table 8 Normalized values for security in RATs.

\begin{tabular}{lllll}
\hline & Encryption & Complexity & Response time & $N S E_{j}$ \\
\hline LTE & $\sim 1.5$ & $\sim 2$ & $\sim 2$ & $\sim 0.4$ \\
GSM & $\sim 2$ & $\sim 3.5$ & $\sim 3$ & $\sim 0.5$ \\
LEO & $\sim 3.5$ & $\sim 3$ & $\sim 3$ & $\sim 0.6$ \\
\hline
\end{tabular}

These values depends to the RATs characters and may varied.

While $R B S_{\text {num }}^{M H Z}$ is the number of minimum packet unit number that can be allocated to a user at a sub time slots, $t s_{\text {num }}$ is the number of sub time slots which makes a time slot, $\lambda_{i}=D_{i}^{g}$ which is the data generating time for node type $i$.

\section{Numerical Results and Discussion}

The weight of data rate, delay, reliability and security for different SG node are calculated or weighted by using Eqs. (4)-(8) and Tables (1)-(8). Figs. 3 and 4 show the data rate and delay normalized value respectively for different type of the SG node over three different RATs that their characteristics were explained in previous section. Fig. 3 shows data rate normalized value for different node types in SG. May the normalized value for low data rate SG nodes are very small in the figure. As it can be seen, WASA delay normalized value is negative in case of using satellite communication RATs and GSM since their latency is higher than WASA delay sensitivity and as it was explained they will be ignored as the RATs candidates to support WASA. Its negative value by using Eq. (5.2) can be seen in Fig. 4. It should be mentioned in the defined CF, the CFs for all RATs have its highest value when CFs are achieved by assigning all of the certain SG node type to the certain RATs (worst case scenario). This achieved CF is the best achieved value for assigning the certain percentage of the mention nodes to different RATs to make an efficient Het-Net. In this case at least the minimum assigning percentage of a certain node to the highest priority RAT can be defined. The CF also shows the fitness degree between node needs and RAT characteristics that was undefined so far.

It is shown in Fig. 4, the nodes like PHEV with lower delay sensitivity have lower delay normalized value than the other high delay sensitive SG nodes.

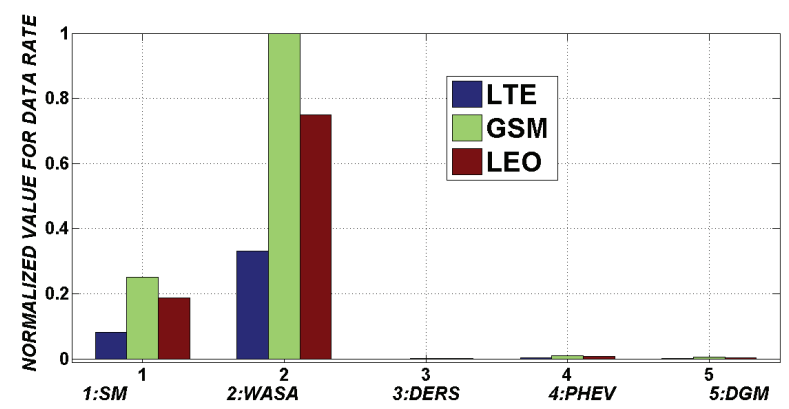

Fig. 3 Data rate normalized value.

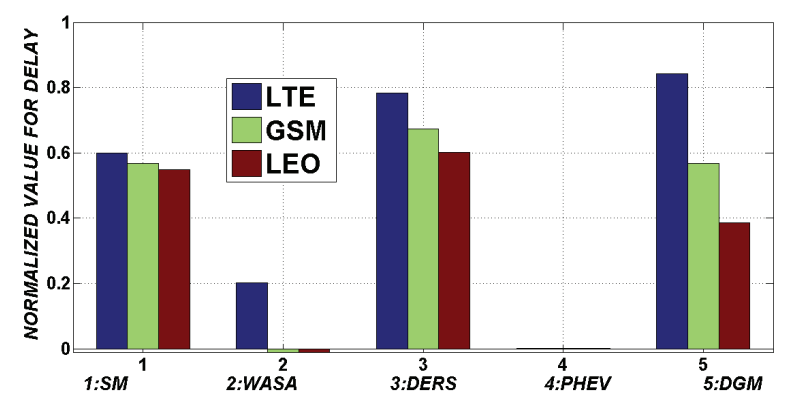

Fig. 4 Delay normalized value.

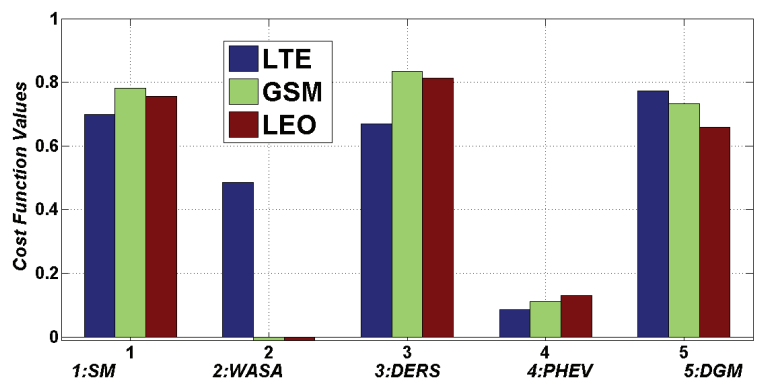

Fig. 5 CF value for different type of the SG node over 3 RATs. 
Based on the achieved CF numerical value for each type of SG node type and the certain RATs which are used in this paper, and the SG user prioritization, the priority table of each certain branch type of the node types in SG are achieved and shown in Fig. 5. The SG nodes (in the two first column of Table 1) have been ordered from up to down based on their priority of respecting the SG goals described in Section 2. LTE due to its higher spectral efficiency is a suitable choice in sense of the SG node type data rate but its CF value does not have a significant difference with the other RATs when it is compared with DERS. Again, as it was discussed 2 second delay is totally acceptable for SMs and assigning the SMs to the LTE with just 10-20 ms causes to have almost high CF. Satellite access technology using DVB-S2 protocol in which the direct end to end communication has been established between terrestrial station and its intrinsic latency is higher than LTE thus, the CF difference between two mentioned RATs is not too much. For the GSM, as it can be seen in Table 8 and Fig. 2, its high RTT (processing and routing delay) makes it more matched with SMs delay but due to high data rate requirement of AMI and the lowest spectral efficiency in GSM, it is worth case for AMI. The reliability and security issues make LTE more suitable although, again, its low latency makes it non-suitable RAT for AMI because AMI can be fulfilled even by much more higher RAT latency. LEO is better than GSM to support AMI because of its higher spectral efficiency. For WASA, as its delay sensitivity is very high, just LTE intrinsic latency can fulfill its delay requirements among the other available RATs. Thus, all traffic generated by WASA must be supported by LTE. As among the available RATs the GSM is not a good RAT in sense of spectral efficiency comparing with two other RATs, may it seems not to be the best option for DERS and DGM but by using the introduced method, it is the best choice for PHEV after LTE because PHEV communication requirements, lowest delay sensitivity, has the highest fitting delay normalized value with GSM (assumed RTT) latency. Due to the same reason, the satellite RAT is the best choice after GSM. PHEV nodes due to its specific communication requirements namely, data rate equals $100 \mathrm{kbps}$ that is somehow high and its delay sensitivity, $5 \mathrm{~s}$ which is the highest delay among the other SG node types, a clear tradeoff for different available RATs CF has been shown. LEO is the best choice for DGM because satellite communication has a high spectral efficiency level and in trade of with its middle range delay sensitivity, GSM is its second priority and LTE is its last priority. As it can be seen, the communication requirements of all SG node type are fulfilled and a trade of among all communication criteria to choose the best RATs for the SG nodes types are done based on an appropriate CF using SG node type communication requirements and RATs communication requirements. Then the matching degree of the RATs communication characteristics and the SG node type communication requirements are evaluated. The SG node prioritization in combination with results of the $\mathrm{CF}$ is shown in Table 9. Plenty of design policies can be done based on this table. In case of spectrum lacking of a certain RATs, the RATs spectrum assignment can be done based on the priority table in Table 9.

Moreover, the introduced method helps to make a Het-Net to support heterogeneous users. The percentages of assigning the numbers of a certain SG node type to the different RATs can be achieved by using an appropriate $\mathrm{CF}$ which includes and considers all RATs communication characteristics and SG node communication requirements matching degree. This $\mathrm{CF}$ in combination with Eb/N0 concept will help to

Table 9 Priority table.

\begin{tabular}{lllll}
\hline & Nodes and priorities & First & Second & Third \\
\hline 1 & WASA & LTE & - & - \\
2 & SM & LTE & LEO & GSM \\
3 & DGM & LEO & GSM & LTE \\
4 & DERS & LTE & LEO & GSM \\
5 & PHEV & LTE & GSM & LEO \\
\hline
\end{tabular}


elaborate a Het-Net based on communication tradeoffs in which $\mathrm{Eb}$ is energy per bits, N0 is the noise spectral density.

\section{Conclusions and Future Work}

Finding a way to allocate the spectrum dynamically as the scarce resources to fulfill all SG node type communication requirements in an efficient way is a significant research challenge. An elaborated method was introduced and investigated to properly choose the best RATs considering their communication characteristics for SG different branch of the node types with different communication requirements while all node types meet their communication necessities in an efficient way in combination with SG node prioritization. The numerical results show how useful this method is while giving a good insight to the SG network designer.

\section{Acknowledgement}

This work is partially supported by the EU ARTEMISJU/CALL2012 project: “ARROWHEAD". Besides, the author thanks Prof. R. Olsen for assistance with the MMPR concept which was defined by him. He helped me during my abroad period at Aalborg University. Also thanks Profs. A. Vanelli-Coralli and D. Tarchi for their great supports that significantly enriched my research activities.

\section{References}

[1] Kouhdaragh, V., Coralli, A. V., and Tarchi, D. 2016. "Using a Cost Function to Choose the Best Communication Technology for Fulfilling the Smart Meters Communication Requirements." In Proceedings of the 1st EAI International Conference on Smart Grid Inspired Future Technologies, 33-42.

[2] Kouhdaragh, V., Tarchi, D., Coralli, A. V., and Corazza, G. E. 2016. "A Cost Function Based Prioritization Method for Smart Grid Communication Network." In Proceedings of the 1st EAI International Conference on Smart Grid Inspired Future Technologies, 16-24.

[3] Kouhdaragh, V., Tarchi, D., Coralli, A. V., and Corazza, G. E. 2015. "Smart Meters Density Effects on the Number of Collectors in a Smart Grid." In Proceedings of the 2015 European Conference on Networks and Communications, 476-81.

[4] Pedersen, A. B., Hauksson, E. B., Andersen, P. B., Poulsen, B., Traeholt, C., and Gantenbein, D. 2010. "Facilitating a Generic Communication Interface to Distributed Energy Resources: Mapping IEC 61850 to RESTful Services." In Proceedings of the 2010 First IEEE International Conference on Smart Grid Communications, 61-6.

[5] Sauter, M. 2011. From GSM to LTE: An Introduction to Mobile Networks and Mobile Broadband, 1st ed. Wiley Journal, Southern Gate, Chichester, West Sussex.

[6] U.S. Department of Energy. 2010. Communications Requirements of Smart Grid Technologies. Technical report.

[7] Mäder, A., Rost, P., and Staehle, D. 2011. "The Challenge of M2M Communications for the Cellular Radio Access Network." 11th Würzburg Workshop on IP: Joint ITG and Euro-NF Workshop "Visions of Future Generation Networks", 525-49.

[8] Shawky, A., Olsen, R., Pedersen, J., and Schwefel, H. P. 2012. "Class-Based Context Quality Optimization for Context Management Frameworks." In Proceedings of the 21st International Conference on Computer Communications and Networks, 1-5.

[9] Kuzlu, M., Pipattanasomporn, M., and Rahman, S. 2014. "Communication Network Requirements for Major Smart Grid Applications in HAN, NAN and WAN." Computer Networks 67 (July): 74-88.

[10] Xiao, Y. 2012. Communication and Network in Smart Grid. University of Alabama, CRC Press, ISBN 9781439878736.

[11] Rengaraju, P., Lung, C.-H., and Srinivasan, A. 2012. "Communication Requirements and Analysis of Distribution Networks Using WiMAX Technology for Smart Grids." In Proceedings of the 8th International Wireless Communications and Mobile Computing Conference, 666-70.

[12] Friedman, N. R. 2002. Distributed Energy Resources Interconnection Systems: Technology Review and Research Needs. National Renewable Energy Laboratory Technical report, URL: http://www.nrel.gov/docs/fy02osti/32459.pdf.

[13] Serrador, A., and Correia, L. M. 2011. "A Cost Function Model for CRRM over Heterogeneous Wireless Networks." Wireless Personal Communications 59 (2): 313-29.

[14] Dahlman, E., Parkvall, S., and Skold, J. 2013. 4G: LTE/LTE-Advanced for Mobile Broadband. 2nd ed., Burlington, MA: Academic Press.

[15] Ivanov, A. 2012. TD-LTE and FDD-LTEA Basic Comparison. Ascom Technical report, 
URL:http://www.ascom.com/en/tems-fdd-lte-vs-td-lte-12. pdf.

[16] Nadia, A., and Aditya, S. K. 2013. "Performance Analysis of GSM Coverage Considering Spectral Efficiency, Interference and Cell Sectoring." International Journal of Engineering and Advanced Technology 2 (4): 115-9.

[17] Goyal, R., Kota, S., Jain, R., Fahmy, S., Vandalore, B., and Kallaus, J. 1998. "Analysis and Simulation of Delay and Buffer Requirements of Satellite-ATM Networks for TCP/IP Traffic." OSU Technical report., http://www.cse.wustl.edu/ jain/papers/ftp/satdelay.pdf.

[18] Kota, S. L., Pahlavan, K., and Leppänen, P. A. 2004. "Performance Analysis of TCP over Satellite ATM." In Broadband Satellite Communications for Internet Access, Springer US, 355-74.

[19] Piemontese, A., Modenini, A., Colavolpe, G., and Alagha, N. 2013. "Improving the Spectral Efficiency of Nonlinear Satellite Systems through Time-Frequency Packing and Advanced Processing." IEEE Transactions on Communications 61 (8): 3404-12.

[20] Ciet, M., Neve, M., Peeters, E., and Quisquater, J. 2003. "Parallel FPGA Implementation of RSA with Residue Number Systems." In Proceedings of the IEEE
International Midwest Symposium on Circuits and Systems, 806-10.

[21] Chen, H.-H., Sharif, H. R., and Nishantha, G. G. D. 2014. Security and Communication Networks. Journal Citation Reports (C Ranking: 2014: 54/77 (Telecommunications); 100/139 (Computer Science Information Systems) Online ISSN: 1939-0122.

[22] Metke, A. R., and Ekl, R. L. 2010. "Security Technology for Smart Grid Networks." IEEE Transactions on Smart Grid 1 (1): 99-107.

[23] URL for related issues to security: http://onlinelibrary.wiley.com/journal/10.1002/\%28ISSN \%291939-0122/earlyview.

[24] Brookson, C. How secure is LTE? ETSI technical report.

[25] Nadeem, A., and Javed, M. Y. 2005. "A Performance Comparison of Data Encryption Algorithms." In Proceedings of the 1st International Conference on Information and Communication Technologies, 84-9.

[26] Solanki, M., Salehi, S., and Esmailpour, A. 2013. "LTE Security: Encryption Algorithm Enhancements." 2013 ASEE Northeast Section Conference Norwich University Reviewed Paper.

[27] http://www.tutorialspoint.com/gsm/gsm_security.htm, GSM-SECURITY AANND EENNCCRRYYPPTTIION. 